\title{
Ectoparasitic mites, ticks and lice of certain domestic birds at Gharbiya Governorate
}

\author{
A. M. Metwally ${ }^{1}$, W. F. Ahmed ${ }^{2}$ and N. S. Amer ${ }^{2, *}$ \\ ${ }^{1}$ Department of Zoology and Nematology, Faculty of Agriculture, Al-Azhar University, Cairo, Egypt \\ ${ }_{2}$ Department of Biological and Environmental Sciences, Faculty of Home Economics, Al-Azhar University, \\ Tanta, Egypt \\ * Corresponding author E-mail: nahedsalahel-sayedamer@azhar.edu.eg (N. Amer).
}

\begin{abstract}
Domestic birds are considered as one of the important sources of food for humans. The parasites generally caused the poor health and decrees in production of these birds, therefore this study was conducted to identify the ectoparasites that infect domestic birds at Gharbia Governorate. During the two successive years (2018 and 2019), several parasites of domestics bird species were collected by Tullgeren funnel. Five domestic bird species, (chickens, ducks, geese, pigeon and turkey hen) at four districts (Al Mahalla Al Kobra, Kutour, Samannoud and Tanta) at Gharbiya governorate were investigated. The collected parasites were forty-four mite species belonging to twentythree families and two suborders, nine tick species belonged to two families and one species and one suborder. Eleven lice species belonged to four families and two suborders. In conclusion, 45 mite species, 9 ticks species and 5 lice species were collected from litter, chickens, ducks, Pekeeny ducks, quails, geese, pigeon in Gharbiya Governorate during successive two years. Ectoparasites are generally considered as the primary cause of many deleterious effects for many poultry species. Such effects including poor health, low growth, and decrease the productivity in Egyptian governorate.
\end{abstract}

Keywords: Ectoparasite; Chicken; Domestic birds.

\section{INTRODUCTION}

Poultry products are considered as one of the most important sources of food for humans, worldwide (Obiora, 1992). Poultry is the most kept livestock and almost every household in villages has about 5-20 indigenous chicken reared under free range management system (Ikpeze et al., 2009). Ectoparasites are generally considered as the primary cause of poor health conditions, growth retardations and decrease in production in local chickens (Chege et al., 2014).

The Poultry industry occupies an important position in the provision of animal protein (meat and egg) to man and generally plays a vital role in the national economy as a revenue provider. Poultry is one of the most intensively reared of the domestic species and one of the most developed and profitable animal production enterprises (Ebrahimi et al., 2016). This study aims to identify the ectoparasites that infect domestic birds at Gharbia governorate

\section{MATERIALS AND METHODS}

Incidence of mites associated with domestic birds including feathers, skins, nasal cavity and feces. A general survey covered the most Gharbiya governorates collected from, Al Mahalla Al Kobra, Kutour, Samannoud and Tanta. A total of sample (400) of these locations.
These field trails started during the two years (2017 and 2018) at Gharbiya governorate.

\section{Collecting sample}

Domestic birds were collected from poultry markets, poultry farms and domestic yards.

Feces were collected from poultry farms and domestic yards.

\section{Transferred samples to the laboratory}

Birds were freshly transferred to the laboratory for parasites extraction. They must be examined immediately or after $30-60$ minutes of hunting. Necessary information including; host, date and locality were recorded.

Feces of birds were placed in paper bags.

\section{Extraction of the mites}

Feathers were removed from the body of birds and feces were put in modified Tullgren funnels for 24 hours. Each funnel has 40 - watt electric lamb according to Krantz and Walter (2009). Adult mites and their stages were removed by a camel hair brush No. (00) or by dissecting needles. Mites were transferred to Petri-dishes (diameter, $9 \mathrm{~cm}$, Hight; $1.5 \mathrm{~cm}$ ) filled with water with airing of Vaseline to prevent escape of mites. Extracted contents of 
the Petri-dishes were examined under the stereoscopic binocular.

Birds skins were carefully examined by stereomicroscope.

Skin mites were collected by specific needle.

\section{Preparing mites}

Collected mites were cleared in Nesbitt's solution. Extracted mites were mounted in Hoyer's medium on glass slides. After that these slides were heated over a hot plate at about $40^{\circ} \mathrm{C}$ to clear. This makes the legs and chelicerae completely stretched. Labels included (species host, date and locality of collection) were fixed to the slides.

\section{RESULTS AND DISSECTION}

\section{Mites associated with domestic bird species}

The collected mites belonging to both Superorder Astigmata and Gamasida:

\section{Superorder Acaridida}

This Superorder was represented by 19 familiesincluding Histostomatidae, Hemisarcoptidae, Euglycyphagidae, Aeroglyphidae, Sudasiidae, Glycyphagidae, Syringobiidae, Apioacaridae, Xolagidae, Dermoglyphidae, Pyroglyphidae, Psoroptodidae, Psoroptidae, Avenzoariidae, Analgidae, Proctophylloididae, Epidormoptidae, Laminosioptidae and Knemidocoptidae, these families have 34 species (Table1).

\section{Family Histostomatidae}

This family was represented by one species, Fibulanoetus sp isolated from feathers of pigeon collected from Tanta with moderate numbers.

\section{Family Hemisarcoptidae}

This family was represented by one species, Hemisarcoptes sp extracted from feathers of ducks and chicken collected from Mahalla Al Kobra and Kutour during summer season with moderate numbers.

\section{Family Euglycyphagidae}

This family was represented by one species, Euglycyphagus intercalates intercalates extracted from feathers of chicken collected from Samannoud during summer season with moderate numbers.

\section{Family Aeroglyphidae}

This family was included one species, Aeroglyphus robustus extracted from feathers of chicken, ducks collected from Tanta with moderate numbers.

\section{Family Sudasiidae}

This family included one species, Suidasia ponifica extracted from chicken collected from Kutour with moderate numbers.

\section{Family Glycyphagidae}

This family was represented by one species, Glycyphagus sp extracted from tissues of chicken and ducks collected from Kutour and Tanta with moderate numbers.

\section{Family Syringobiidae}

This family was represented by two species, Phyllochaeta sp and Syringophilus africanus extracted from tissues of pigeon and geese collected from Kutour, Samannoud and Tanta with moderate numbers.

\section{Family Apioacaridae}

This family included one species, Atelepoda sp extracted from feathers of ducks and geese collected from Kutour and Tanta with moderate numbers.

\section{Family Xolagidae}

This family included one species, Vingrassie $\mathrm{sp}$ were extracted from feathers of ducks, chicken and turkey-hen collected from Kutour and Mahalla Al Kobra with moderate numbers.

\section{Family Dermoglyphidae}

This family was represented by five species, Dermoglyphus columbae and D. micocera extracted from skin of pigeon while D. elongates, D. farina, and Dermoglyphus sp were extracted from skin of ducks, geese and chicken collected from Kutour, Mahalla Al Kobra, Samannoud and Tanta with moderate numbers.

\section{Family Pyroglyphidae}

This family was represented by one species, Pyroglyphus arfricanus extracted from skin of chicken and pigeon collected from Kutour and, Mahalla Al Kobra with moderate numbers.

\section{Family Psoroptodidae}

This family was represented by one species, Pandalura strigisoti extracted from skin of chicken collected from Kutour and Mahalla Al Kobra with moderate numbers.

\section{Family Psoroptidae}

This family included one species, Psoroptes cunniculi extracted from skin of chicken 
collected from Tanta, Kutour and Mahalla Al Kobra with moderate numbers.

\section{Family Avenzoariidae}

This family included two species, Scutamegininia sp and Bdelordychus sp extracted from feathers of ducks collected from Samannoud and Mahalla Al Kobra with moderate numbers.Family Analgidae

This family was represented by three species, Analges spiniger and A. leiopus extracted from feathers of chicken while, Meginia columbae extracted from feathers of pigeon and chicken collected from Samannoud and Mahalla Al Kobra with great numbers.

\section{Family Proctophylloididae}

This family was represented by two species, Proctophylloderus oriantalis and Pterophagus striculus extracted from feathers of chicken collected from Samannoud, Kutour and Mahalla Al Kobra with moderate numbers with great numbers.

\section{Family Epidormoptidae}

This family was represented by four species, Epidermoptes perdicola, Epidermoptes sp, $M$. anchor and Myialges sp. were extracted from skin of pigeon while, the species $M$. anchor and Myialges sp extracted only from skin of ducks, chicken and turkey-hen collected from Tanta, Samannoud, Kutour and Mahalla Al Kobra with great numbers.

\section{Family Laminosioptidae}

This family included three species, Laminosioptes cysticola, L. hymenopterus and Laminosioptes sp extracted from skin of pigeon and turkey-hen collected from Tanta, Kutour and Mahalla Al Kobra with great numbers.

\section{Family Knemidocoptidae}

This family was represented by two species, kenemidocoptes sp and Neonemidocoptes gallina extracted from skin of chicken collected from Tanta, Kutour and Mahalla Al Kobra with moderate numbers.

\section{Superorder Gamasida}

This Superorder was represented by four families; Dermanyssidae, Macronyssidae, Rhinonyssidae and Feryanidae these families have 10 species (Table1).

\section{Family Dermanyssidae}

This family was represented by one species, Dermanyssus gallinae isolated from blood sucking mites of pigeon, ducks and chicken collected from Tanta by a few numbers during the summer season (2017) only.

\section{Family Macronyssidae}

This family was included three species, Ornithonyssus bursa, Ornithonyssus sylviarium and $O$. hoogstraoli extracted from skin as blood sucking mites of turkey-hen and pigeon collected from Mahalla Al Kobra and Kutour during summer season with moderate numbers.

\section{Family Rhinonyssidae}

This family was included five species, Rhinonyssus colymbicola, Rhinonyssus caledonicus, Rhinonyssus bisetosus, Neoryssus columbea and Sternostoma framcheacolam extracted from nasal cavity of pigeon, ducks and turkey-hen collected from Samannoud, Mahalla Al Kobra Kutour and Tanta during summer season with moderate numbers.

\section{Family Feryanidae}

This family was represented by one species, Freyana largifolia extracted from nasal cavity of ducks collected from Kutour and Tanta.

Incidence of ixodid ticks associated with some domestic bird species at Gharbiya governorate.

\section{Family Ixodidae Canestrini}

This family was represented by five species belonging to two genera as,

\section{Genus Hayalomma}

This genus was included three species as, Hayalomma impltatum, $H$. marginatum and $H$. dromedari isolated from skin of turkey-hen, pigeon and ducks collected from Kutour and Mahalla Al Kobra with few numbers (Table 2).

\section{Genus Rhipicephalus}

This genus was represented by two species; Rhipicephalus turanicus and $R$. guihoni isolated from skin of pigeon and ducks collected from Kutour and Mahalla Al Kobra with few numbers (Table 2).

\section{Incidence of argasid tick associated with some domestic bird species at Gharbiya governorate.}

\section{Family Argasidae Canestrini}

This family was represented by four species; Argas persicus, A. hermanni, A. streptopelian and 
Ornithodorinae coniceps isolated from skin of turkey-hen, ducks, pigeon and chicken collected from Tanta, Kutour and Mahalla Al Kobra with few numbers (Table 3).

\section{Ectoparasitic lice associated with Domestic} bird species at Gharbiya governorate

\section{Order Mallbophaga}

This order was represented by two suborders. Amblycera: this superorder includes three families: Menoponidae, Ricinidae and Laemobothiridae. Ischcera: this superorder has one family incloud, Philopteridae (Table 4).

\section{Family Menoponidae}

This family included three species, Colpcephalus helzeenthali, Menopos sp and Mumidicola sp extracted during the year (2017 and 2018) from skin of ducks, and turkey-hen collected from Kutour and Mahalla Al Kobra with a high number.

\section{Family Ricinidae}

This family included one species, Ricinus $\mathrm{sp}$ isolated with few numbers from skin of chicken and pigeon from Tanta during the period of study.

\section{Family Laemobothiridae}

This family was included one species; Laemobothrion $\mathrm{sp}$ isolated with a high number from skin of chicken, pigeon and ducks from Kutour during the period of study.

\section{Family Philopteridae}

This family was represented by six species; Philopterus residus, Columbicola bacillus, C. columbe L, C. baculoides, Gonoides sp and Heptapsogaster sp isolated from skin and tissues of Pigeon, chicken, and ducks collected from Kutour, Samannoud and Mahalla Al Kobra with a high number.

Our study revealed that the presence of parasitic found on domestic birds. Number of mite species were recorded in Gharbiya governorate was 2 suborders, 23 families and 44 species. These mites were previously audited by many investigators, Fain and Philips (1977), Fain et al. (1977), Rakha (1980), El Kammah et al. (1982), Hoogstraal (1984), El Kammah et al, (1990), Abd-Allah (1993), Fain and drugmand (1993), Gaud (1996), Fan (2000), Fan and Zhang (2004), El Kammah (2007) and Abdel-Gawad (2008).

Also, the parasitic ticks were recorded on domestic birds were 1 family, 2 genera and 5 species. The obtained data agree with Hoogstraal (1956), Taylor et al. (1966), El Kammah et al. (1982), Hoogstraal (1984), El Kammah et al. (1990), El Kammah (2007) and Guglielmone et al. (2010).

On the other hand, lice were recorded as parasitic on domestic birds were 1 order, 2 suborders, 4 families and 11 species. Our data agree with Emerson (1972), Rekasi (1979), Honacki (1982), Lyal (1985) and Beaucournu (1986)

\section{CONCLUSION}

In conclusion, 45 mite species, 9 ticks species and 5 lice species were collected from litter, chickens, ducks, pekeeny ducks, quails, geese, pigeon in Gharbiya Governorate during successive two years. Ectoparasites are generally considered as the primary cause of many deleterious effects for many poultry species. Such effects including poor health, low growth, and decrease the productivity in Egyptian governorate.

\section{REFERENCES}

Abd-Allah, A.A., 1993. Studies on Some Mites Inhabiting Wild Birds. MSc. Thesis, Fac. Agric. Al-Azhar Univ., 209 pp.

Abdel-Gawad, A.M., 2008. Studies on Some Mites Associated with Some Birds in Egypt. PhD. Thesis, Zoology Department. Fac. Scien. Al-Azhar Univ. (Girls). 424 pp.

Beaucournu, J.C., Aubert, M.F.A., 1986. A propos des Mallophagous de carnivores savages en France (Mallophagous: Trichodectiidae). Bull. Soc. Franc. Parasitol., 4, 273-277.

Chege, H.W., Kemboi, D.C., Bebora, L.C., Maingi, N., Nyaga, P.N., Mbuthia, P.N., Njagi, P.G., Githinji, J., 2014. Chicken parasites and local treatments used against them in Mbeere District, Kenya. Livest. Res. Rural. Dev., 26, 2528.

Ebrahimi, M.H., Samiei, M., Anousheh, K., Razi Jalali, D., 2016. Identification of ectoparasites in indigenous poultry in southern areas of West Azerbaijan, Iran: A study on the prevalence and importance of these parasites. Archives of Razi Institute, Vol. 71 (4), 253-258.

El Kammah, K.M., 2007. Tick and parasitic. Fac. Agri. Cairo University, 153pp.

El Kammah, K.M., Hamdy, B.H., Saoudi, O., 1982. The influence of ectoparasitic (ticks and mites) on host serum enzymes. J. Egypt. Soc. Parasitol. 12 (1), 71-79.

El Kammah, K.M., Oyom, L.M.L., Madbouly, M.H., Habeeb, S.M., 1990. Morphological description of the chicken mite Ornithonyssus bursa (Berlese, 1888) (Dermanyssidae) from Egypt. Bull. Soc. Ent. Egypt. 69, 295-304.

Emerson, K.C., 1972. Checklist of the Mallophagous of North America (North of Mexico). Part 1. Suborder 
Ischnocera. Deseret Test Center, Dugway Proving Ground. Dugway. Utah. 200 pp.

Fain, A., drugmand, D., 1993. Notes on the genus Hexatbrombium Cooreman 1944 (Acari: Trombididae) with description of a few tribe and species from Afrotropical staphylinidae (coleoptera). Bull. Ann. Soc. Roy. Belg. Entomol. 129, 121-128.

Fain, A., Philips, J.R., 1977. Astigmatic mites from nests of birds of prey in U.S.A., description of four new species of Glycyphagidae. Internat. J. Acarol. 3, 105- 14.

Fain, A., Hyland, k.e., Aitken, T.H.G., 1977. Flower mites of the family Ascidae phoretic in nasal cavities of birds (Acarina: Mesostigmata) Acta. Zool. Pathol. Atverp. 69, 99-154.

Fan, Q.H., 2000. The morphology of Xenocaligonellidus smileyi (Acari: Xenocaligonellidae). In systematic and faunistic research on Chinese insects, ed. B. K. Zhang Yalin. 290-297.

Fan, Q.H., Zhang, Z.Q., 2004. Revision of Rhizoglyphus Claparede (Acari: Acaridae) of Australia and Oceania. London.

Gaud, J., 1996. Feather mites of the world (Acarina: Astigmata). The superspacific taxa. Ann. Mus. Royal Afr. Cent. Sci. Zool., 277, 1-436.

Guglielmone A.A., Robbins, R.G., Apanasevich, D.A., Petney, T.N., Pena, A.E., Horak, I.G., Shaa, R., Barker, S.C., 2010. The Argasidae, Ixodidae and Nuttalliae (Acari: Ixodidae) of the world. A list of valid species names. Zootaxa, 2528, 1-28.

Honacki, J.H., 1982. Mammal species of the world. Ataxonomic and geographic reference. Lawrence.
Kansas: Allen Press and the Association of Systematic Collection. 496 pp.

Hoogstraal, H., 1956. African Ixodidae and Ticks of the Sudan. Bur. Med. Surg. U.S. Navy 1, 1101pp.

Hoogstraal, H., 1984. Ticks and mites in. disease transmission. Hunter's tropical medicine, by G. T. Strickland 6. Ed., W. B. Saunders Co., Philadelphia, P. A. Chap. 101, 896-903.

Ikpeze, O.O., Amagba, I.C., Eneanya, C.I., 2009. Preliminary survey of ectoparasites of chicken in Awka, south-eastern Nigeria. Animal Research International 5 (2), 848-851.

Krantz, G.W., Walter, D.E., 2009. A manual of Acarology. Texas Tec University. Third ed. 808 pp.

Lyal, C.H.C., 1985. A Cladistic analysis and classification of trichodectid mammal lice (Phthiraptera: Ischnocera). Bulletin of the British Museum. Natural History (Entomology). 51, 187-346.

Obiora, F.C., 1992. A Guide to Poultry Production in the Tropics, Acena Publishers, Enugu, Nigeria, 1st edition.

Rakha, M.A., 1980. Taxonomical And Biological Studies on Some Astigmatid Mites of Birds. PhD. Thesis, Fac. Agric. Cairo Univ. Egypt., 384 pp.

Rekasi, J., 1979. The Mallophaga collection of the Hungarian Natural History Museum. II. Parasitology Hungarica. 12, 93-98.

Taylor, R.M., Hurlbut, H.S., Work, T.H., Kingston, J.R., Hoogstraal, H., 1966. Arboviruses isolate from Argas ticks in Egypt: Quaranfil, Chenuda, and Nyamanini. Amer. J. Trop. Med. Hyg. 15, 76-86. 
Table 1. Incidence of mites associated with some domestic bird species at Gharbiya governorate.

\begin{tabular}{|c|c|c|c|c|c|c|}
\hline \multicolumn{3}{|r|}{ Mites } & \multicolumn{4}{|c|}{ Domestic Birds } \\
\hline 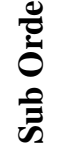 & 虔 & Mite species & $\begin{array}{l}\text { English } \\
\text { Name }\end{array}$ & $\begin{array}{c}\text { Examined } \\
\text { material }\end{array}$ & Abundance & Locality \\
\hline \multirow{6}{*}{ 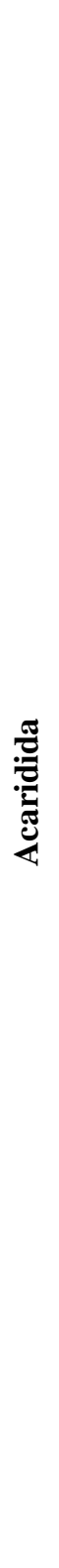 } & 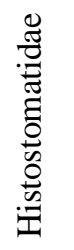 & Fibulanoetus sp & Pigeon & Feathers & ++ & Tanta \\
\hline & 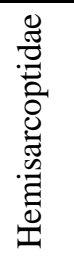 & Hemisarcoptes sp & $\begin{array}{l}\text { ducks, } \\
\text { chicken } \\
\text { and } \\
\text { pigeon }\end{array}$ & Feathers & ++ & $\begin{array}{l}\text { Mahalla Al } \\
\text { Kobra and } \\
\text { Kutour }\end{array}$ \\
\hline & 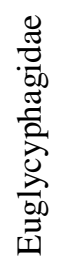 & $\begin{array}{l}\text { Euglycyphagus intercalates } \\
\text { (Fain and Philips) }\end{array}$ & $\begin{array}{l}\text { chicken, } \\
\text { ducks } \\
\text { pigeon }\end{array}$ & Feathers & ++ & Samannoud \\
\hline & 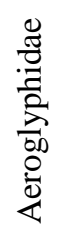 & Aeroglyphus robustus (Banks) & $\begin{array}{l}\text { Chicke, } \\
\text { ducks } \\
\text { Pigeon }\end{array}$ & Feathers & ++ & Tanta \\
\hline & 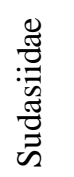 & Suidasia ponifica (Oudemans) & $\begin{array}{l}\text { ducks, } \\
\text { chicken } \\
\text { and } \\
\text { pigeon }\end{array}$ & Tissues & ++ & Kutour \\
\hline & 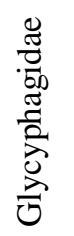 & Glycyphagus sp & $\begin{array}{l}\text { chicken } \\
\text { and } \\
\text { ducks }\end{array}$ & Tissues & ++ & $\begin{array}{l}\text { Kutour } \\
\text { and Tanta. }\end{array}$ \\
\hline
\end{tabular}




\section{Continue: Table 1}

\begin{tabular}{|c|c|c|c|c|c|c|}
\hline \multicolumn{3}{|r|}{ Mites } & \multicolumn{4}{|c|}{ Domestic Birds } \\
\hline $\begin{array}{l}\vdots \\
0 \\
0 \\
0 \\
0 \\
0 \\
0\end{array}$ & 를 & Mite species & $\begin{array}{l}\text { English } \\
\text { Name }\end{array}$ & $\begin{array}{l}\text { Examined } \\
\text { material }\end{array}$ & Abundance & Locality \\
\hline \multirow{6}{*}{ 苞 } & $\begin{array}{l}0 \\
\pi \\
: 0 \\
: 0 \\
0 \\
0 \\
0 \\
0 \\
\text { क }\end{array}$ & $\begin{array}{l}\text { Phyllochaeta sp } \\
\text { Syringophilus africanus } \\
\text { (Hughes) }\end{array}$ & $\begin{array}{l}\text { Pigeon } \\
\text { and } \\
\text { geese }\end{array}$ & Tissues & +++ & $\begin{array}{l}\text { Kutour, } \\
\text { Samannoud } \\
\text { and Tanta }\end{array}$ \\
\hline & 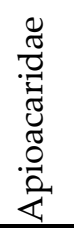 & Atelepoda sp & $\begin{array}{l}\text { ducks, } \\
\text { geese } \\
\text { and } \\
\text { pigeon }\end{array}$ & Feathers & ++ & $\begin{array}{l}\text { Kutour } \\
\text { and Tanta. }\end{array}$ \\
\hline & $\begin{array}{l}\frac{\pi}{\pi} \\
\frac{\pi}{60} \\
\frac{\pi}{0} \\
\times\end{array}$ & Vingrassie sp & $\begin{array}{l}\text { ducks, } \\
\text { chicken, } \\
\text { turkey- } \\
\text { hen and } \\
\text { pigeon }\end{array}$ & Feathers & ++ & $\begin{array}{l}\text { Kutour and } \\
\text { Mahalla Al } \\
\text { Kobra }\end{array}$ \\
\hline & 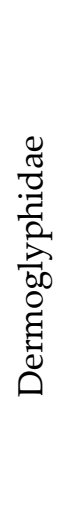 & $\begin{array}{l}\text { Dermoglyphus elongates } \\
\text { (Megnin) } \\
\text { Dermoglyphus columbae } \\
\text { (Sugimotos) } \\
\text { Dermoglyphus farinae } \\
\text { (Hushes and Seoidi) } \\
\text { Dermoglyphus micocera (Gaud) } \\
\text { Dermoglyphus sp }\end{array}$ & $\begin{array}{l}\text { ducks, } \\
\text { geese, } \\
\text { chicken } \\
\text { and } \\
\text { pigeon }\end{array}$ & Skin & +++ & $\begin{array}{l}\text { Kutour, } \\
\text { Mahalla Al } \\
\text { Kobra, } \\
\text { Samannoud } \\
\text { and Tanta }\end{array}$ \\
\hline & $\begin{array}{l}0 \\
\frac{\pi}{0} \\
0 \\
2 \\
2 \\
000 \\
0 \\
0 \\
0\end{array}$ & $\begin{array}{l}\text { Pyroglyphus arfricanus } \\
\text { (Hugos) }\end{array}$ & $\begin{array}{l}\text { chicken } \\
\text { and } \\
\text { pigeon }\end{array}$ & Skin & ++ & $\begin{array}{l}\text { Kutour } \\
\text { and } \\
\text { Mahalla } \\
\text { Al Kobra }\end{array}$ \\
\hline & $\begin{array}{l}0 \\
\pi \\
0 \\
0 \\
0 \\
0 \\
0 \\
0 \\
0 \\
0\end{array}$ & Pandalura strigisoti (Buchholz) & $\begin{array}{l}\text { chicken } \\
\text { and } \\
\text { pigeon }\end{array}$ & Skin & ++ & $\begin{array}{l}\text { Kutour and } \\
\text { Mahalla Al } \\
\text { Kobra }\end{array}$ \\
\hline
\end{tabular}




\begin{tabular}{|c|c|c|c|c|c|c|}
\hline \multicolumn{3}{|r|}{ Mites } & \multicolumn{4}{|c|}{ Domestic Birds } \\
\hline $\begin{array}{l}\frac{t}{0} \\
0 \\
0 \\
0 \\
0 \\
0\end{array}$ & 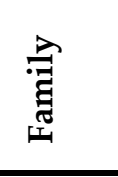 & Mite species & $\begin{array}{c}\text { English } \\
\text { Name }\end{array}$ & $\begin{array}{l}\text { Examined } \\
\text { material }\end{array}$ & Abundance & Locality \\
\hline \multirow{6}{*}{ : } & 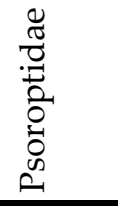 & Psoroptes cunniculi (Delatand) & $\begin{array}{l}\text { chicken } \\
\text { and } \\
\text { ducks }\end{array}$ & Skin & ++ & $\begin{array}{l}\text { Kutour and } \\
\text { Mahalla Al } \\
\text { Kobra }\end{array}$ \\
\hline & 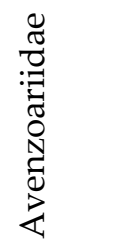 & $\begin{array}{l}\text { Scutamegininia sp } \\
\text { Bdelordychus sp }\end{array}$ & $\begin{array}{l}\text { chicken, } \\
\text { ducks, } \\
\text { geese } \\
\text { and } \\
\text { pigeon }\end{array}$ & Feathers & ++ & $\begin{array}{l}\text { Samannoud } \\
\text { and Mahalla } \\
\text { Al Kobra. }\end{array}$ \\
\hline & 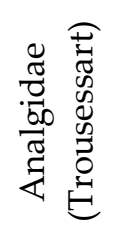 & $\begin{array}{l}\text { Analges spiniger (Giebel) } \\
\text { Analges leiopus (Gaad and } \\
\text { Mouchet) } \\
\text { Meginia columbae (Boscholz) }\end{array}$ & $\begin{array}{l}\text { chicken, } \\
\text { ducks } \\
\text { and } \\
\text { pigeon }\end{array}$ & Feathers & +++ & $\begin{array}{l}\text { Samannoud } \\
\text { and Mahalla } \\
\text { Al Kobra. }\end{array}$ \\
\hline & 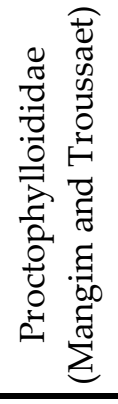 & $\begin{array}{l}\text { Proctophylloderus oriantalis } \\
\text { (Gaud) } \\
\text { Pterophagus striculus } \\
\text { (Megmin) }\end{array}$ & $\begin{array}{l}\text { chicken, } \\
\text { ducks } \\
\text { and } \\
\text { pigeon }\end{array}$ & Feathers & +++ & $\begin{array}{l}\text { Samannoud, } \\
\text { Kutour and } \\
\text { Mahalla Al } \\
\text { Kobra. }\end{array}$ \\
\hline & 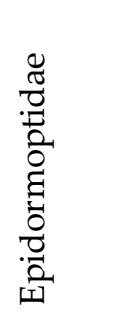 & $\begin{array}{l}\text { Epidermoptes perdicola (Fain } \\
\text { and Evans) } \\
\text { Epidermoptes sp } \\
\text { Myialges anchora (Trouessart) } \\
\text { Myialges sp }\end{array}$ & $\begin{array}{l}\text { chicken, } \\
\text { turkey- } \\
\text { hen, } \\
\text { ducks } \\
\text { and } \\
\text { pigeon }\end{array}$ & Skin & +++ & $\begin{array}{l}\text { Tanta, } \\
\text { Samannoud, } \\
\text { Kutour and } \\
\text { Mahalla Al } \\
\text { Kobra. }\end{array}$ \\
\hline & 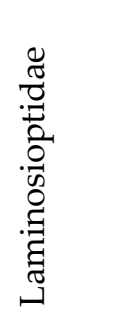 & $\begin{array}{l}\text { Laminosioptes hymenopterus } \\
\text { (Jones and Goud) } \\
\text { Laminosioptes crysticola } \\
\text { (Vizioli) } \\
\text { Lamiosptes sp }\end{array}$ & $\begin{array}{l}\text { chicken, } \\
\text { turkey- } \\
\text { hen, } \\
\text { ducks, } \\
\text { geese } \\
\text { and } \\
\text { pigeon }\end{array}$ & Skin & +++ & $\begin{array}{l}\text { Tanta, } \\
\text { Kutour }\end{array}$ \\
\hline
\end{tabular}




\section{Continue: Table 1.}

\begin{tabular}{|c|c|c|c|c|c|c|}
\hline \multicolumn{3}{|r|}{ Mites } & \multicolumn{4}{|c|}{ Domestic Birds } \\
\hline $\begin{array}{l}\frac{0}{0} \\
0 \\
0 \\
0 \\
0 \\
5\end{array}$ & 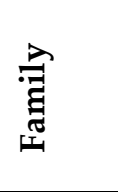 & Mite species & $\begin{array}{c}\text { English } \\
\text { Name }\end{array}$ & $\begin{array}{c}\text { Examined } \\
\text { material }\end{array}$ & Abundance & Locality \\
\hline$\frac{\pi}{0}: \frac{\pi}{0}$ & 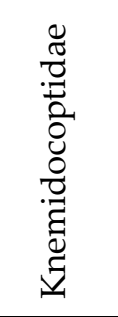 & $\begin{array}{l}\text { Kenonemidocoptes sp } \\
\text { Neonemidocoptes gallina } \\
\text { (Bailliet) }\end{array}$ & $\begin{array}{l}\text { chicken, } \\
\text { ducks } \\
\text { and } \\
\text { pigeon }\end{array}$ & Skin & +++ & $\begin{array}{l}\text { Tanta, } \\
\text { Kutour and } \\
\text { Mahalla Al } \\
\text { Kobra }\end{array}$ \\
\hline & 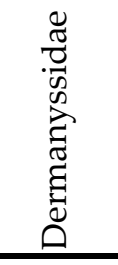 & Dermanyssus gallinae (Degeer) & ppigeon & Blood & ++ & Tanta \\
\hline & 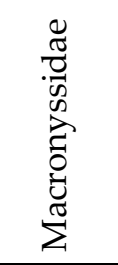 & $\begin{array}{l}\text { Ornithonyssus bursa (Berlese) } \\
\text { Ornithonyssus sylviarium } \\
\text { (Canestrini and Fanzago) } \\
\text { Ornithonyssus sp }\end{array}$ & $\begin{array}{l}\text { turkey- } \\
\text { hen, } \\
\text { ducks } \\
\text { and } \\
\text { pigeon }\end{array}$ & Blood & +++ & $\begin{array}{l}\text { Mahalla Al } \\
\text { Kobra and } \\
\text { Kutour }\end{array}$ \\
\hline 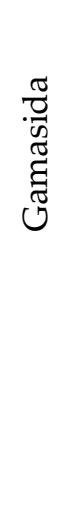 & 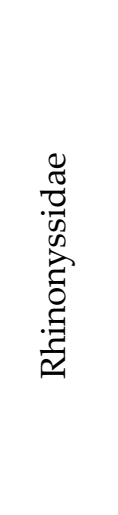 & $\begin{array}{l}\text { Rhinonyssus colymbicola (Fain } \\
\text { and Bafrot) } \\
\text { Rhinonyssus caledonicus } \\
\text { (Hirst) } \\
\text { Rhinonyssus bisetosus } \\
\text { (Stranitman) } \\
\text { Neoryssus columbea } \\
\text { (Crosseley) } \\
\text { Sternostoma framcheacolam }\end{array}$ & $\begin{array}{l}\text { turkey- } \\
\text { hen, } \\
\text { ducks, } \\
\text { geese } \\
\text { and } \\
\text { pigeon }\end{array}$ & $\begin{array}{l}\text { nasal } \\
\text { cavit and } \\
\text { Tissue }\end{array}$ & +++ & $\begin{array}{l}\text { Samannoud } \\
\text {, Mahalla Al } \\
\text { Kobra } \\
\text { Kutour and } \\
\text { Tanta }\end{array}$ \\
\hline & 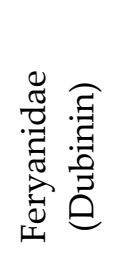 & $\begin{array}{l}\text { Freyana largifolia } \\
\text { (Megnin and trouessart) }\end{array}$ & $\begin{array}{l}\text { ducks } \\
\text { and } \\
\text { pigeon }\end{array}$ & $\begin{array}{l}\text { nasal } \\
\text { cavity } \\
\text { and } \\
\text { Tissue }\end{array}$ & ++ & $\begin{array}{l}\text { Kutour and } \\
\text { Tanta }\end{array}$ \\
\hline
\end{tabular}

$+=$ Few number $\quad+=$ Moderate numbers $\quad++=$ Great numbers 
Table 2. Incidence of Ixodid ticks associated with some domestic bird species at Gharbiya governorate.

\begin{tabular}{|c|c|c|c|c|c|c|}
\hline \multicolumn{3}{|r|}{ Ixodid ticks } & \multicolumn{4}{|c|}{ domestic birds } \\
\hline 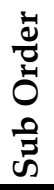 & 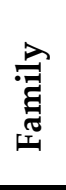 & Species & $\begin{array}{l}\text { English } \\
\text { Name }\end{array}$ & $\begin{array}{c}\text { Examined } \\
\text { material }\end{array}$ & Abundance & Locality \\
\hline \multirow[t]{2}{*}{ 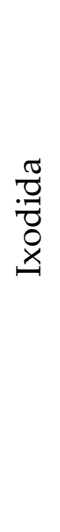 } & \multirow{2}{*}{ 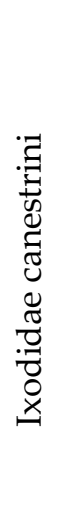 } & $\begin{array}{l}\text { Hayalomma impltatum } \\
\text { (Schizel and Schlottes) } \\
\text { Hayalomma marginatum } \\
\text { Hayalomma dromedari (Toch) }\end{array}$ & $\begin{array}{l}\text { turkey- } \\
\text { hen, } \\
\text { pigeon } \\
\text { and } \\
\text { ducks }\end{array}$ & $\begin{array}{l}\text { Skin and } \\
\text { blood }\end{array}$ & ++ & $\begin{array}{l}\text { Kutour } \\
\text { and } \\
\text { Mahalla } \\
\text { Al } \\
\text { Kobra }\end{array}$ \\
\hline & & $\begin{array}{l}\text { Rhipicephalus turanicus } \\
\text { (Pomerantzov) } \\
\text { Rhipicephalus guihoni } \\
\text { (Morel and Vassiliades) }\end{array}$ & $\begin{array}{l}\text { pigeon } \\
\text { and } \\
\text { ducks }\end{array}$ & Feathers & ++ & $\begin{array}{l}\text { Kutour } \\
\text { and } \\
\text { Mahalla } \\
\mathrm{Al} \\
\text { Kobra }\end{array}$ \\
\hline
\end{tabular}

Table 3. Incidence of Argasid tick associated with some domestic bird species at Gharbiya governorate:

\begin{tabular}{|c|c|c|c|c|c|c|}
\hline \multicolumn{3}{|r|}{ Ixodid ticks } & \multicolumn{4}{|c|}{ domestic birds } \\
\hline 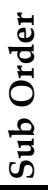 & 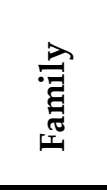 & Species & $\begin{array}{l}\text { English } \\
\text { Name }\end{array}$ & $\begin{array}{c}\text { Examined } \\
\text { material }\end{array}$ & Abundance & Locality \\
\hline 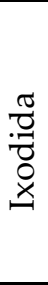 & 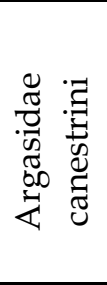 & $\begin{array}{l}\text { Argas persicus (Oken) } \\
\text { Argas hermanni (Audokin) } \\
\text { Argas streptopelian } \\
\text { (Hoogestrol and Hornes) } \\
\text { Ornithodorus coniceps } \\
\text { (Canestrini) }\end{array}$ & $\begin{array}{l}\text { turkey- } \\
\text { hen, } \\
\text { ducks, } \\
\text { pigeon } \\
\text { and } \\
\text { chicken }\end{array}$ & skin & ++ & $\begin{array}{l}\text { Tanta, } \\
\text { Kutour } \\
\text { and } \\
\text { Mahalla } \\
\text { Al Kobra }\end{array}$ \\
\hline
\end{tabular}

$+=$ Few number $\quad++=$ Moderate numbers $\quad++=$ Great numbers 
Table 4. Incidence of lice associated with some domestic bird species at Gharbiya governorate: -

\begin{tabular}{|c|c|c|c|c|c|c|c|}
\hline \multicolumn{4}{|c|}{ Ixodid ticks } & \multicolumn{4}{|c|}{ domestic birds } \\
\hline ț & \begin{tabular}{l}
$\vdots$ \\
\multirow{0}{0}{} \\
0 \\
0 \\
0 \\
0
\end{tabular} & 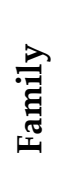 & Lice species & $\begin{array}{c}\text { English } \\
\text { Name }\end{array}$ & $\begin{array}{l}\text { Examined } \\
\text { material }\end{array}$ & Abundance & Locality \\
\hline \multirow{4}{*}{ 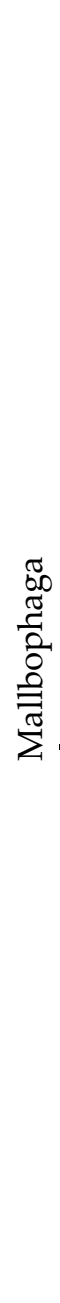 } & \multirow{3}{*}{ 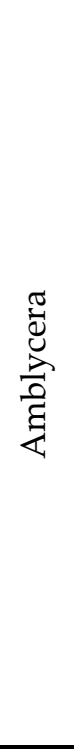 } & 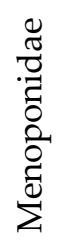 & $\begin{array}{l}\text { Colpcephalus } \\
\text { helzeenthali } \\
\text { Menopos sp } \\
\text { Mumidicola sp }\end{array}$ & $\begin{array}{l}\text { ducks, } \\
\text { and } \\
\text { turkey- } \\
\text { hen }\end{array}$ & Skin & +++ & $\begin{array}{l}\text { Kutour and } \\
\text { Mahalla Al } \\
\text { Kobra }\end{array}$ \\
\hline & & 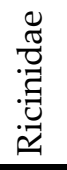 & Ricinus sp & $\begin{array}{l}\text { chicken } \\
\text { and } \\
\text { Pigeon }\end{array}$ & Skin & + & Tanta \\
\hline & & 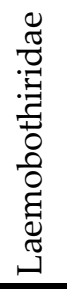 & Laemobothrion sp & $\begin{array}{l}\text { chicken, } \\
\text { pigeon } \\
\text { and } \\
\text { ducks }\end{array}$ & Feathers & ++ & Kutour \\
\hline & 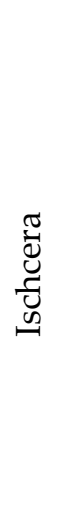 & 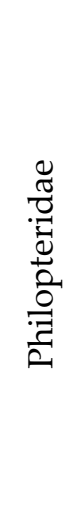 & $\begin{array}{l}\text { Philopterus residus } \\
\text { (Ziotorzycka) } \\
\text { Columbicola bacillus } \\
\text { (Giebl) } \\
\text { Columbicola columbe L. } \\
\text { Columbicola baculoides } \\
\text { (Paine) } \\
\text { Gonoides oustralis } \\
\text { Heptapsogaster sp }\end{array}$ & $\begin{array}{l}\text { Pigeon, } \\
\text { chicken, } \\
\text { and } \\
\text { ducks }\end{array}$ & Feathers & +++ & $\begin{array}{l}\text { Kutour, } \\
\text { Samannoud } \\
\text { and Mahalla } \\
\text { Al Kobra }\end{array}$ \\
\hline
\end{tabular}

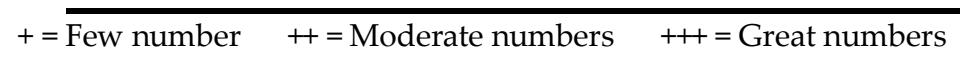




\section{تواجد الحلم والقراد والقمل المثطفل خارجيا على بعض الطيور الداجنة بمافظة الغربية

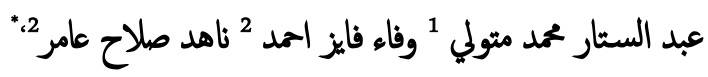

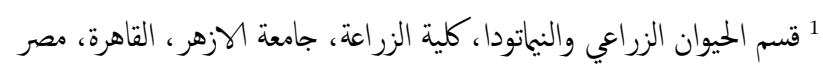

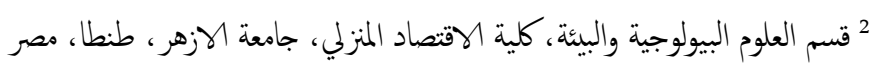

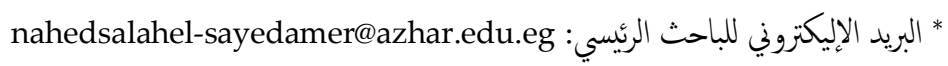

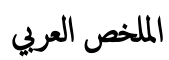

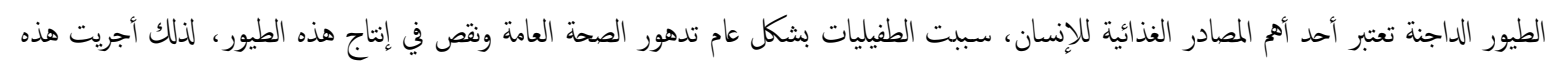

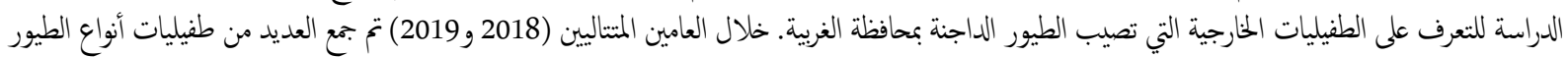

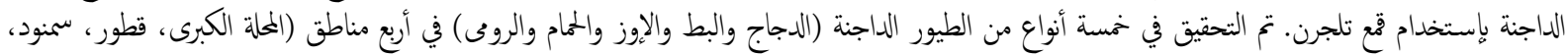

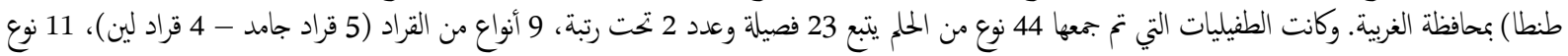

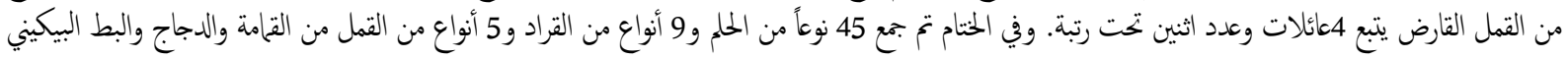

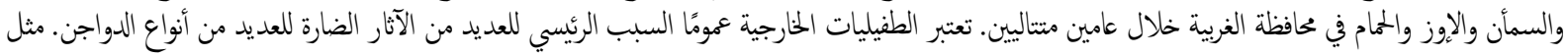

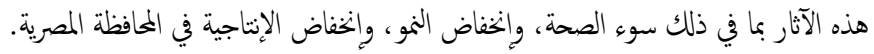
الكلمات المفتاحية: الطفيليات الخارجية، دجاج، الطيور الداجنة. 